\title{
ON THE VALIDITY OF WILSON'S APPROACH TO GENERAL RELATIVITY
}

\author{
R. RIETH \\ Max-Planck-Gesellschaft, AG-Gravitationstheorie \\ Friedrich-Schiller-Universität, D-07743 Jena, Germany \\ E-mail: ror@gravi.physik.uni-jena.de
}

\begin{abstract}
The Wilson scheme and the Einstein dynamics are compared for binary systems. At the second post-Newtonian approximation, genuine two-body aspects are found to differ by up to $114 \%$.

1. Introduction. Recently Wilson and Mathews [1] proposed a truncated version of the Einstein field equations to treat the coalescence of binary neutron stars in a much simplified but still sufficiently precise manner. The main idea of this approximation is to neglect the independent ("true") degrees of freedom of the gravitational field, i.e. in particular, the full gravitational radiation content. For spherically symmetric processes the proposed scheme is identical with the Einstein equations, in non-spherically symmetric dynamical situations, even stationary ones, the proposed scheme and the Einstein theory only coincide at the first post-Newtonian level of approximation. It is perhaps worth mentioning that, in contrast to the Einstein theory, the proposed scheme should allow post-Newtonian series expansions to all orders in (integer) powers of $1 / c^{2}$.

Wilson et al. [2] have applied this scheme to the question of instabilities in close neutron star binaries and found the remarkable result that general relativity may cause otherwise stable stars to collapse prior to merging. In another recent paper Cook et al. [3] tested the Wilson scheme for isolated, rapidly rotating relativistic stars. They found a deviation from the Einstein theory of at most $5 \%$ which they interpreted as very encouraging for a better understanding of binary star evolution.

In this article the Wilson scheme is applied to point-like binary star systems at the second post-Newtonian approximation and the periastron advance as well as the orbital period are calculated. For circular motion, also the dependence of the angular momentum on the orbital angular frequency is given. The obtained results are confronted with the corresponding results of the Einstein dynamics.
\end{abstract}

1991 Mathematics Subject Classification: 83C25.

The paper is in final form and no version of it will be published elsewhere. 
2. Second post-Newtonian Wilson scheme and Einstein dynamics. In the Einstein theory the periastron advance and the orbital period have been calculated by Damour and Schäfer [4] starting from a second post-Newtonian Hamilton function. In isotropic coordinates and in the center of mass system $\left(\mathbf{P}_{1}=-\mathbf{P}_{2}=\mathbf{P}\right)$, in reduced varibales $(\mathbf{p}=\mathbf{P} / \mu, \mathbf{r}=\mathbf{R} / G M)$, the reduced Hamilton function $\hat{H}=H / \mu$ reads,

$$
\begin{aligned}
\hat{H}(\mathbf{r}, \mathbf{p}, \nu) & =\frac{1}{2} \mathbf{p}^{2}-\frac{1}{r}-\frac{1}{8 c^{2}}(1-3 \nu) \mathbf{p}^{4}-\frac{1}{2 r c^{2}}\left[(3+\nu) \mathbf{p}^{2}+\nu(\mathbf{n} \cdot \mathbf{p})^{2}\right] \\
& +\frac{1}{2 r^{2} c^{2}}+\frac{1}{16 c^{4}}\left(1-5 \nu+5 \nu^{2}\right) \mathbf{p}^{6} \\
& +\frac{1}{8 r c^{4}}\left[\left(5-20 \nu-3 \nu^{2}\right) \mathbf{p}^{4}-2 \nu^{2} \mathbf{p}^{2}(\mathbf{n} \cdot \mathbf{p})^{2}-3 \nu^{2}(\mathbf{n} \cdot \mathbf{p})^{4}\right] \\
& +\frac{1}{2 r^{2} c^{4}}\left[(5+8 \nu) \mathbf{p}^{2}+3 \nu(\mathbf{n} \cdot \mathbf{p})^{2}\right]-\frac{1}{4 r^{3} c^{4}}(1+3 \nu),
\end{aligned}
$$

where $\nu=\mu / M$ with $\mu=M_{1} M_{2} / M$ and $M=M_{1}+M_{2} . M_{1}$ and $M_{2}$ denote the masses of the two bodies. The linear momenta of the bodies are $\mathbf{P}_{1}$ and $\mathbf{P}_{2}$, and $\mathbf{R}$ denotes a difference of their coordinate position vectors, $\mathbf{R}=\mathbf{R}_{1}-\mathbf{R}_{2} ; c$ is the velocity of light.

The fractional periastron advance per orbital revolution, $k$, and the orbital period, $P$, were found to be

$$
\begin{gathered}
k=\frac{3}{h^{2} c^{2}}\left[1+\frac{1}{2}(5-2 \nu) \frac{E}{c^{2}}+\frac{5}{4}(7-2 \nu) \frac{1}{h^{2} c^{2}}\right], \\
P=\frac{2}{\pi G M} \sqrt{-2 E}^{3}\left[1-\frac{1}{4}(15-\nu) \frac{E}{c^{2}}-\frac{3}{32}\left(35+30 \nu+3 \nu^{2}\right) \frac{E^{2}}{c^{4}}+\frac{3}{2}(5-2 \nu) \frac{\sqrt{-2 E}^{3}}{h c^{4}}\right],
\end{gathered}
$$

where $E$ is the total center-of-mass energy (numerical value of $\hat{H}$ ) and where $h$ is the absolute value of the reduced angular momentum $\mathbf{J} / G M \mu$.

The Hamilton function in the Wilson scheme is easily obtained as two-body special case of the n-body matter Hamilton function $H_{\text {mat }}$ of Schäfer [5] (eq. (3.14)). In reduced form this Hamiltonian reads (isotropic coordinates),

$$
\begin{aligned}
\hat{H}_{m a t}(\mathbf{r}, \mathbf{p}, \nu) & =\frac{1}{2} \mathbf{p}^{2}-\frac{1}{r}-\frac{1}{8 c^{2}}(1-3 \nu) \mathbf{p}^{4}-\frac{1}{2 r c^{2}}\left[(3+\nu) \mathbf{p}^{2}+\nu(\mathbf{n} \cdot \mathbf{p})^{2}\right]+\frac{1}{2 r^{2} c^{2}} \\
& +\frac{1}{16 c^{4}}\left(1-5 \nu+5 \nu^{2}\right) \mathbf{p}^{6}+\frac{5}{8 r c^{4}}(1-4 \nu) \mathbf{p}^{4} \\
& +\frac{1}{4 r^{2} c^{4}}\left[(10+19 \nu) \mathbf{p}^{2}-3 \nu(\mathbf{n} \cdot \mathbf{p})^{2}\right]-\frac{1}{4 r^{3} c^{4}}(1+\nu) .
\end{aligned}
$$

In the test-body limit, $\nu=0$, the two Hamilton functions $\hat{H}$ and $\hat{H}_{\text {mat }}$ coincide as they should because of spherical symmetry of the central body.

The periastron advance of the dynamics eq. (4) is easily obtained following the path way of Ref. [4]. It comes out in the form

$$
k_{\text {mat }}=\frac{3}{h^{2} c^{2}}\left[1+\frac{1}{2}\left(5-\frac{3}{2} \nu-\frac{41}{12} \nu^{2}\right) \frac{E}{c^{2}}+\frac{1}{4}\left(35-\frac{27}{2} \nu-\frac{31}{4} \nu^{2}\right) \frac{1}{h^{2} c^{2}}\right] .
$$

As one can see from equations (2) and (5) the two different periastron advances have 
the following structure

$$
k=\frac{1}{c^{2}} k_{o}^{1 p N}+\frac{1}{c^{4}}\left(k_{o}^{2 p N}+k_{\nu}^{2 p N}\right)
$$

where $k_{o}^{n p N}$ denotes the $\nu$-independent terms and $k_{\nu}^{n p N}$ the $\nu$-dependent terms of $k . k$ and $k_{\text {mat }}$ are different in the $\nu$-dependent terms of the second post-Newtonian order only. Using the Newtonian relation between energy and angular momentum, $E=\left(e^{2}-1\right) / 2 h^{2}$, where $e$ denotes the eccentricity of the binary orbit, for the same energy and angular monentum, the fractional difference between the two periastron advances at the genuine two-body $2 \mathrm{pN}$ level reads

$$
\Delta k=\left(k-k_{m a t}\right) /\left|k_{\nu}^{2 p N}\right|=\frac{1}{24\left(4+e^{2}\right)}\left[4(12+13 \nu)-(6-41 \nu) e^{2}\right] .
$$

The application of this expression to the case of equal-mass binaries $(\nu=1 / 4)$ in circular orbits $(e=0)$ gives a fractional difference between $k$ and $k_{m a t}$ at the genuine two-body $2 \mathrm{pN}$ level of about $63 \%$.

The orbital period in the Wilson scheme is obtained in the form, again following the route of Ref. [4],

$$
\begin{aligned}
P_{\text {mat }}=\frac{2 \pi G M}{\sqrt{-2 E}^{3}}[1 & -\frac{1}{4}(15-\nu) \frac{E}{c^{2}}-\frac{15}{32}\left(7+6 \nu-25 \nu^{2}\right) \frac{E^{2}}{c^{4}} \\
& \left.+\frac{1}{2}\left(15-\frac{9}{2} \nu-\frac{41}{4} \nu^{2}\right) \frac{\sqrt{-2 E}^{3}}{h c^{4}}\right] .
\end{aligned}
$$

Analogously to the periastron advance, the two orbital periods have the following structure

$$
P=P^{N}+\frac{1}{c^{2}}\left(P_{o}^{1 p N}+P_{\nu}^{1 p N}\right)+\frac{1}{c^{4}}\left(P_{o}^{2 p N}+P_{\nu}^{2 p N}\right) .
$$

Also they are different in the $\nu$-dependent terms of the second post-Newtonian order only. For the same energy and angular momentum, the fractional difference between the two orbital periods at the genuine two-body $2 \mathrm{pN}$ level reads

$$
\Delta P=\left(P-P_{\text {mat }}\right) /\left|P_{\nu}^{2 p N}\right|=-\frac{16}{3} \frac{6-\nu\left(41-24 \sqrt{1-e^{2}}\right)}{128+3(10+\nu) \sqrt{1-e^{2}}},
$$

where we have used again the Newtonian relation between energy and angular momentum. In the case of $e=0$ and $\nu=1 / 4$, the expression (10) reaches $6 \%$.

For circular orbits the angular frequency, $\omega$, is defined through the expression $\Phi / P$, where $\Phi$, the angle advance for one orbital period, is given by $\Phi=2 \pi(1+k)$. Taking into account the relation between the energy and the angular momentum for circular orbits, for our two dynamical situations, Einstein and Wilson respectively, the relations between angular momentum and orbital angular frequency turn out to be

$$
\begin{gathered}
h=\frac{1}{\omega^{1 / 3}}\left[1+\frac{1}{2}\left(3+\frac{1}{3} \nu\right) \frac{\omega^{2 / 3}}{c^{2}}+\frac{1}{8}\left(27-19 \nu+\frac{1}{3} \nu^{2}\right) \frac{\omega^{4 / 3}}{c^{4}}\right], \\
h_{\text {mat }}=\frac{1}{\omega^{1 / 3}}\left[1+\frac{1}{2}\left(3+\frac{1}{3} \nu\right) \frac{\omega^{2 / 3}}{c^{2}}+\frac{1}{8}\left(27-39 \nu-\frac{17}{3} \nu^{2}\right) \frac{\omega^{4 / 3}}{c^{4}}\right] .
\end{gathered}
$$


The fractional difference between $h$ and $h_{\text {mat }}$ at the genuine two-body $2 \mathrm{pN}$ level reads, applying the same frequency in both cases,

$$
\Delta h=\left(h-h_{m a t}\right) /\left|h_{\nu}^{2 p N}\right|=6 \frac{10+3 \nu}{57-\nu} .
$$

For the case of equal masses, $\nu=1 / 4, \Delta h$ amounts to $113.7 \%$.

3. Conclusion. In the test-body limit as well as in the first post-Newtonian approximation the calculated expressions for the periastron advance, the orbital period, and the angular momentum coincide. They only differ in the genuine binary $2 \mathrm{pN}$ parts, this means in the $\nu$-dependent terms of $2 \mathrm{pN}$ order. The fractional difference of the $2 \mathrm{pN}$ $\nu$-dependent terms for $h$ takes the remarkably large value of about $114 \%$ in the case of equal-mass binaries in circular motion having the same orbital frequency. Therefore, if genuine two-body aspects play a significant role in the general relativistic dynamics, the Wilson scheme can not be expected to approximate general relativity reasonably well.

Acknowledgments. The author thanks Gerhard Schäfer for many valuable discussions.

\section{References}

[1] J. R. Wilson, in: Texas Symposium on 3-Dimensional Numerical Relativity, edited by Matzner (University of Texas, Austin, Texas, 1990); J. R. Wilson and G. J. Mathews, in: Frontiers in Numerical Relativity, edited by C. R. Evans, L. S. Finn., and D. W. Hobill (Cambridge University Press, Cambridge, England, 1989), pp. 306-314.

[2] J. R. Wilson and G. J. Mathews, Phys. Rev. Lett. 75, 4161 (1995); J. R. Wilson, G. J. Mathews and P. Marronetti, Phys. Rev. D 54, 1317 (1996).

[3] G. B. Cook, S. L. Shapiro, and S. A. Teukolsky, Phys. Rev. D 53, 5533 (1996).

[4] T. Damour and G. Schäfer, Nuovo Cimento B 101, 127 (1988).

[5] G. Schäfer, Ann. Phys. (N.Y.) 161, 81 (1985). 